\title{
Electron-phonon interaction on the surface of a three-dimensional topological insulator
}

\author{
V. Parente, ${ }^{1,2,4}$ A. Tagliacozzo, ${ }^{1,2}$ F. von Oppen, ${ }^{3}$ and F. Guinea ${ }^{4}$ \\ ${ }^{1}$ Dipartimento di Fisica, Università di Napoli Federico II, Via Cintia, I-80126 Napoli, Italy \\ ${ }^{2}$ CNR-SPIN, Monte Sant'Angelo-Via Cintia, I-80126 Napoli, Italy \\ ${ }^{3}$ Dahlem Center for Complex Quantum Systems and Fachbereich Physik, Freie Universität Berlin, 14195 Berlin, Germany \\ ${ }^{4}$ Instituto de Ciencia de Materiales de Madrid (CSIC), Sor Juana Inés de la Cruz 3, Madrid 28049, Spain
}

(Received 29 May 2013; revised manuscript received 27 July 2013; published 23 August 2013)

\begin{abstract}
We analyze the interaction at the surface of a three-dimensional (3D) topological insulator among 2D electron states belonging to the Dirac cone close to the point of the Brillouin zone and the Rayleigh surface phonon mode. The model deals with an elastic continuum in the long-wavelength limit, in Random Phase Approximation (RPA). Screening of the electronic polarization is quite effective at small wave vectors. On the other hand, the absence of backscattering for the Dirac electrons at the Fermi surface is partly responsible for the reduced influence of the electron-phonon interaction in renormalizing the phonon dispersion at finite wave vectors. We infer that softening of the Rayleigh mode appears as unlikely, at least for the case of a clean and defect-free surface to which our approximate treatment applies. The dielectric response to virtual excitation of the Rayleigh phonon could drive the electron-electron interaction attractive at low frequencies, but the average weak coupling pairing interaction is found to be too small to induce a surface superconducting instability.
\end{abstract}

DOI: 10.1103/PhysRevB.88.075432 PACS number(s): 72.10.Di, 63.22.-m, 74.90.+n, 85.25.Qc

\section{INTRODUCTION}

Topological insulators (TIs) have attracted a great deal of attention in recent times. ${ }^{1,2}$ Their most significant feature is the existence of topologically protected surface states. In this context, the electron-phonon $(e-p h)$ interaction at the surface of a three-dimensional (3D) TI is particularly relevant, as it could make a significant contribution to the transport properties of the surface states. ${ }^{3-6}$ Experimental evidence is ambiguous. Angleresolved photoemission spectroscopy (ARPES) measurements reveal small effects on surface electrons, ${ }^{6}$ suggesting that there is a negligible interaction between electrons and surface phonons at the boundary of a 3D TI. On the other hand, a strong coupling of phonons with surface electrons is extracted from measurements of surface phonon spectra in $\mathrm{Bi}_{2} \mathrm{Se}_{3}$, leading to a strong Kohn anomaly for transverse optical surface phonons, while there is no evidence of a Rayleigh acoustical branch ${ }^{4}$ which would usually be expected to exist in systems with a free surface. ${ }^{5}$ A possible explanation given for the latter feature is a different coupling between planes in the quintuple layer of $\mathrm{Bi}_{2} \mathrm{Se}_{3}$.

Here we explore a different scenario and assume that the Rayleigh acoustical branch is well defined up to large-enough $\vec{q}$ wave vectors parallel to the surface and we would like to check its renormalization due to the electronic states at the surface. This assumption motivates a study of the electron-phonon interaction at the surface of a 3D TI. Our low-energy theory involves a single Dirac cone at the $\Gamma$ point for surface electrons. The electron screening depends on the $e$-ph matrix element $M_{s s^{\prime} \vec{k}}(\vec{q})$ and the screened electronic polarization operator $\mathcal{P}$. Here $s, s^{\prime}$ are helicity indices and, in principle, the matrix element could be nondiagonal in the helicity. However, we show that, due to the high symmetry of the $\Gamma$ point, up to the linear order in the strain vector $\mathbf{u}$, the main contribution to the $e$-ph interaction is the deformation potential seen by the surface electrons and is diagonal in the helicity (see Appendix A ).
On the other hand, while the bare electronic polarization operator $\mathcal{P}$ may be large even at small $q$ values, the screening in Random-Phase Approximation (RPA) turns out to be very effective up to $q \sim \alpha_{F S} k_{F} / 2 \pi$, which is a transferred momentum spanning a large portion of the Fermi surface. Here $k_{F}$ is the Fermi wave vector, $\alpha_{F S}$ is the effective fine structure constant $\alpha_{F S}=e^{2} / \epsilon \hbar v_{F} \sim 0.1$, and $\epsilon \sim 50$ is the average bulk dielectric constant. ${ }^{7,8}$

It turns out that the crucial quantity that determines the strength of the $e$-ph coupling is the $q=|\vec{q}|$ vector-dependent structure factor $\Lambda_{l}(q)$, quantifying the localization of the phonon mode at the surface and its overlap with the surface electron density. In the ideal long wavelength limit of a continuum medium $\Lambda_{l}(q)$ is quantitatively determined by the typical decay length of the Rayleigh mode away from the surface $\propto 1 / q . \Lambda_{l}(q)$ is larger the smaller is $q$, while it is heavily suppressed at large momenta. It follows that, in this case, a small renormalization of the phonon frequency and of the electronic plasma surface mode is produced.

However, the ideal limit may be questionable, in view of the scattering by impurities and defects at the surface, and we also show that a different $q$ dependence of $\Lambda_{l}(q)$ may be quite critical for the survival of the Rayleigh mode. In this context, we investigate the limiting case $\Lambda_{l}(q) \sim 1$, which makes the reconstruction of the surface possible. Were this the case, STM measurements could reveal the reconstruction of the surface.

In Sec. II we introduce the model and evaluate the matrix element of the $e$-ph interaction. In Sec. III we discuss the damping of the Rayleigh mode, the quality factor, and the remote possibility of a softening of the phonon Rayleigh mode in the continuum model. In Sec. IV the influence of the $e$-ph interaction on the electron-electron $(e-e)$ scattering is analyzed within RPA. At low frequencies, there is a pole in the dielectric constant due to $q$ vectors matching the phonon dispersion, with a change of sign of the $e$-e interaction. In the static limit the sign change survives and allows us to give an estimate of the superconductive pairing interaction. However, a weak 
coupling approach suggests that the Rayleigh mode cannot contribute significantly to the superconducting instability. A short summary and the conclusions are reported in Sec. V.

Appendix A elaborates on the symmetry properties of the $e$-ph coupling Hamiltonian and proves that the occurrence of the Dirac cone at the $\Gamma$ point constrains the $e$-ph interaction to a featureless deformation potential. Appendix B collects results of the polarization operator $P$ for Dirac electrons which are used in RPA. Appendix C develops a perturbative approach in the continuum elastic dynamics of the surface displacements to deal with a strong anisotropy of the elastic constants while approaching the outermost layers of the surface. The derivation shows that, although the sound velocity is softened, the mode remains always well defined.

\section{THE MODEL}

On the free surface of a TI, the Rayleigh surface phonon mode is expected to have a major role in the long wavelength limit, due to its characteristic localization length which is proportional to the inverse of the wave vector of propagation q. In the linear elasticity theory an isotropic continuum with stress-free boundary conditions at the surface, $\sigma_{i j} n_{j}=0(i, j=$ $1,2,3)$, where $\sigma_{i j}$ is the stress tensor and $\mathbf{n}$ is the normal to the surface (taken in the $z$ direction), allows for a lattice displacement field $\mathbf{u}$, of the form

$$
\mathbf{u}(\mathbf{q}, z)=\left(i q \phi_{l}-\frac{d \phi_{t}}{d z}\right) \hat{e}_{q}+\left(\frac{d \phi_{l}}{d z}+i q \phi_{t}\right) \hat{e}_{z} .
$$

Here $\hat{e}_{q}$ is the direction of the wave vector $\mathbf{q}$ and $\hat{e}_{z}$ is in the direction orthogonal to the surface. $\phi_{l}$ and $\phi_{t}$ are the longitudinal and transverse parts, given by

$$
\phi_{l}^{(R)}=\sqrt{\frac{C}{q}} e^{-\lambda_{l} q z}, \quad \phi_{t}^{(R)}=-\sqrt{\frac{C}{q}} \frac{2 i \lambda_{l}}{1+\lambda_{t}^{2}} e^{-\lambda_{t} q z} .
$$

The coefficient $C$ is completely determined by the velocities for longitudinal and transverse phonons $c_{l}=2800 \mathrm{~m} / \mathrm{s}$ and $c_{t}=1600 \mathrm{~m} / \mathrm{s}$, through the dimensionless quantities $\lambda_{l}=$ $\sqrt{1-c_{R} / c_{l}}$ and $\lambda_{t}=\sqrt{1-c_{R} / c_{t}}$,

$$
\frac{1}{C}=\lambda_{l}-\lambda_{t}+\frac{\left(\lambda_{l}-\lambda_{t}\right)^{2}}{2 \lambda_{l}^{2} \lambda_{t}}
$$

and its value for $\mathrm{Bi}_{2} \mathrm{Te}_{3}$ is 1.2.9,10 The Rayleigh mode has a linear dispersion relation $\omega_{q}^{(0)}=c_{R} q$ with velocity $c_{R}=$ $0.89 c_{t}$.

In the following, we focus on the $e$-ph interaction of the Rayleigh mode localized at the surface with the Dirac surface states of the TI.

The surface states are localized at the surface as well, with a decay length inside the insulating bulk $\hbar v_{F} / \Delta$, where $\Delta$ is the gap in the bulk spectrum. In the ideal case, the overlap of the electronic surface density with the wave function of the Rayleigh phonons, in the $z$ direction, is a linear combination of the components of the strain tensor given by

$$
U_{i j}(q)=\frac{2 \Delta}{\hbar v_{F}} \int_{0}^{\infty} d z \frac{\left[\partial_{i} u_{j}(\mathbf{q}, z)+\partial_{j} u_{i}(\mathbf{q}, z)\right]}{2} e^{-2 \Delta z / \hbar v_{F}} .
$$

We consider a low-energy effective Hamiltonian for the surface electronic states close to the $\Gamma$ point in $\vec{k}$ space, $\mathcal{H}=\mathcal{H}_{0}+$ $\mathcal{H}_{e-p h}$, reduced to two dimensions. $\mathcal{H}_{0}=-i \hbar v_{F} \boldsymbol{\sigma} \cdot \nabla$ is the free Dirac Hamiltonian in the surface plane and $\mathcal{H}_{e-p h}$ is the $e$-ph interaction. The second quantized Hamiltonian reads

$$
\begin{aligned}
\mathcal{H}_{0}-\mu \mathcal{N} & =\sum_{k, s= \pm} \epsilon_{k s} c_{k, s}^{\dagger} c_{k, s}, \quad \epsilon_{k s}=s \hbar v_{F}|k|-\mu \\
\mathcal{H}_{e-p h} & =\sum_{\boldsymbol{k}, \boldsymbol{q}, \omega, s, s^{\prime}} M_{s s^{\prime} \vec{k}}(q)\left(a_{q}+a_{q}^{\dagger}\right) c_{\mathbf{k}+\mathbf{q}, s^{\prime}}^{\dagger} c_{\mathbf{k}, s}+\text { H.c. }
\end{aligned}
$$

Here $s, s^{\prime}= \pm$ label the helicity of surface states $|\mathbf{k}, s\rangle, \mu=$ $\hbar v_{F} k_{F}$ is the chemical potential, and $v_{F}$ is the Fermi velocity $\left(v_{F}=4.36 \times 10^{5} \mathrm{~m} / \mathrm{s}\right.$ for $\left.\mathrm{Bi}_{2} \mathrm{Te}_{3}\right)$. The matrix element of the $e$-ph interaction, $M_{s s^{\prime} \vec{k}}(\vec{q})$, can be written in terms of the integrated strain field of Eq. (4) as

$$
M_{s s^{\prime} \vec{k}}(\vec{q})=\alpha \sum_{i j}\left\langle\mathbf{k}+\mathbf{q}, s\left|A_{i j} U_{i j}(q)\right| \mathbf{k}, s^{\prime}\right\rangle .
$$

The constant $\alpha$ characterizes the strength of the $e$-ph coupling. ${ }^{3}$ The matrix elements contain the contraction of the strain tensor with the $4 \times 4$ matrices $A_{i j}$, defined in the orbital $\otimes$ spin space, spanned by the TI two-band model. ${ }^{1}$ The matrices $A_{i j}$ should be invariant with respect to the symmetry operations of the little group ${ }^{11}$ of the wave vectors of the electrons and phonons. Their components in terms of Dirac matrices are listed in Table I of Appendix A, together with the point symmetries that they satisfy. As shown in Appendix A, the high symmetry of the $\Gamma$ point implies that the only possible coupling is with the trace of the strain tensor through the identity matrix $A_{i j}=\delta_{i j}$. It follows that the $e$-ph interaction conserves helicity at $\mathbf{k}$ close to the $\Gamma$ point and is independent of it. Therefore, by dropping the label $s$ henceforth, the matrix element of the $e$-ph interaction reads

$$
M(q)=\alpha \sqrt{\frac{C}{q \mathcal{A}}}\left(\frac{\omega_{q}^{(0)}}{c_{l}}\right)^{2} \sqrt{\frac{\hbar}{2 \rho_{M} \omega_{q}^{(0)}}} \Lambda_{l}(q) .
$$

Here $\mathcal{A}$ is the unit cell area and the estimated value for $\alpha$ for $\mathrm{Bi}_{2} \mathrm{Te}_{3}$ is $35 \mathrm{eV}$, as extracted from first-principles calculations including static screening effects. ${ }^{12}$ The mass density of $\mathrm{Bi}_{2} \mathrm{Te}_{3}$ is ${ }^{3} \rho_{M}=7860 \mathrm{Kg} / \mathrm{m}^{3}$. The actual $q$ dependence of the longitudinal structure factor $\Lambda_{l}(q)$ turns out to be quite critical for the strength of the $e$-ph coupling, as shown in the following. The simplest expression for $\Lambda_{l}(q)$, which takes into account just the localization of the Rayleigh phonon in the integral of Eq. (4), is

$$
\Lambda_{l}(q)=\frac{\Delta}{\Delta+\lambda_{l} \hbar v_{F}|q| / 2} .
$$

$\Lambda_{l}(q)$ attains the largest value $\left[\Lambda_{l}(q) \approx 1\right]$ when $q \ll \Delta / \hbar v_{F}$. Equation (8) does not include effects of surface defects such as edge dislocations or localized scattering centers, which are likely to be present, e.g., due to Te vacancies.

It can be expected that the most relevant transferred momenta $q$ for the $e$-ph interaction are the ones connecting points at the Fermi surface up to $q \sim 2 k_{F}$. Due to the absence of backscattering at the ideal surface of the TI, it turns out that the scattering with small $q$ has a major role. In the following 
we also consider $\Lambda_{l}(q)$ as a given parameter to explore the consequences of the $e$-ph interaction in full.

\section{EFFECTS OF ELECTRONS ON PHONONS}

Electronic screening effects can be taken into account, within RPA. The electronic polarization operator takes the form $^{13}$

$$
\mathcal{P}(q, \omega)=\frac{P(q, \omega)}{1-v_{q} P(q, \omega)},
$$

where $P(q, \omega)$ is the bare polarization operator per unit area and $v_{q}=e^{2} / 2 \epsilon q$ is the 2D electron-electron $(e-e)$ interaction (see Appendix B). ${ }^{14}$ To investigate the effects of electrons on phonons at the surface of a TI, we first study the phonon damping, defined through the imaginary part of (9) defined through the imaginary part of the polarization $\mathcal{P}$ [see Sec. III A]. The real part of (9), on the other hand, determines the correction to the phonon dispersion $\Delta E=|M(q, \omega)|^{2} \operatorname{Re} \mathcal{P}(q, \omega)$ [see Sec. III B].

\section{A. Phonon damping and quality factor}

The phonon damping is calculated as $\Gamma_{q}=$ $|M(q)|^{2} \operatorname{Im} \mathcal{P}(q, \omega)$, where $M(q)$ is the $e$-ph matrix element given in Eq. (7). In the small $q$ limit, with the frequency $\omega$ satisfying the inequalities

$$
\frac{\hbar \omega}{\hbar v_{F} q} \ll 1 \ll \frac{\mu}{\hbar v_{F} q},
$$

the bare electronic polarization operator per unit area, $P(q, \omega)$, has the imaginary part

$$
\operatorname{Im} P(q, \omega)=\frac{1}{4} \frac{\omega}{\hbar v_{F}^{2}} \sqrt{\left(\frac{2 \mu}{\hbar v_{F} q}\right)^{2}-1},
$$

which can be quite large. Within RPA, we obtain for the full polarization operator

$$
\operatorname{Im} \mathcal{P}(q, \omega)= \begin{cases}\frac{\omega}{\hbar v_{F}^{2} \alpha_{F S}} \frac{q}{2 k_{F}} & \text { for } q<\frac{\alpha_{F S} k_{F}}{2 \pi}, \\ \frac{1}{4} \frac{\omega}{\hbar v_{F}^{2}} \sqrt{\left(\frac{2 \mu}{\hbar v_{F} q}\right)^{2}-1} & \text { for } q>\frac{\alpha_{F S} k_{F}}{2 \pi} .\end{cases}
$$

In the limit $q<k_{F}$, the phonon decay rate $\Gamma_{q}$ is

$$
\Gamma_{q}= \begin{cases}\frac{\pi}{8 \alpha_{F S}} K\left(k_{F}\right) \frac{\left(\hbar \omega_{q}^{(0)} \Lambda_{l}(q)\right)^{2}}{\mu} \frac{q^{2}}{k_{F}^{2}}, & \text { for } q<\frac{\alpha_{F S} k_{F}}{2 \pi}, \\ \frac{\pi}{8} K\left(k_{F}\right) \frac{\left(\hbar \omega_{q}^{(0)} \Lambda_{l}(q)\right)^{2}}{\mu} & \text { for } q>\frac{\alpha_{F S} k_{F}}{2 \pi} .\end{cases}
$$

In our case, the effective fine structure constant, $\alpha_{F S}=$ $e^{2} / \epsilon \hbar v_{F}$, is quite large, typically $\sim 0.1$. This implies that the damping remains rather small (of order $q^{2} / k_{F}^{2}$ ) up to relatively high values of $q$. The dimensionless quantity

$$
K\left(k_{F}\right)=\frac{2 \alpha^{2} C}{\pi} \frac{c_{R}^{2}}{v_{F} c_{l}} \frac{k_{F}^{2}}{\hbar \rho_{M} c_{l}^{3}}=2.35 \mathrm{~nm}^{2} k_{F}^{2}
$$

plays a central role in determining the features of the $e$-ph interaction at the surface of the TI. Its value is strongly dependent on the Fermi wave vector. The ratio $c_{R} / v_{F}$ is $\approx 3 \times 10^{-3}$ (Ref. 3). As the linewidth vanishes for $q \rightarrow 0$, the Rayleigh phonons are well defined at small wave vectors. The quality factor $Q=\hbar \omega_{q} / \Gamma_{q}$, measuring the broadening of the phonon energy, is plotted in Fig. 1, also for larger wave

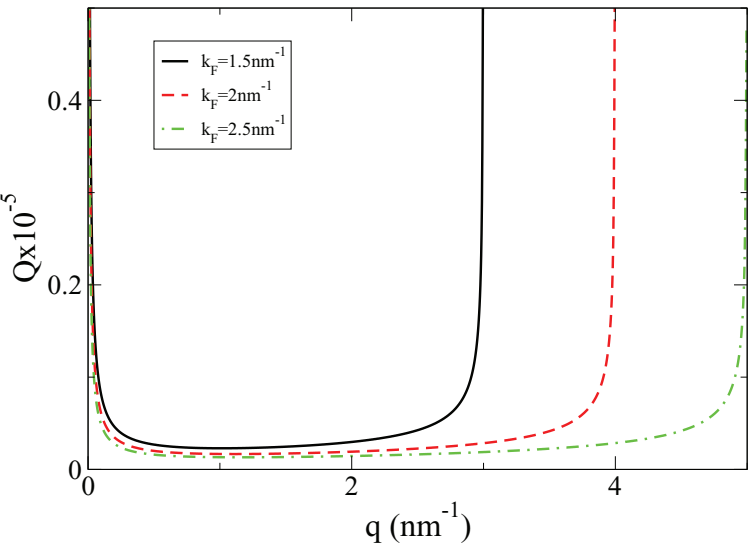

FIG. 1. (Color online) Quality factor $Q$ as a function of momentum $q$ for different values of the Fermi wave vector: $k_{F}=1.5 \mathrm{~nm}^{-1}$ (black solid line), $k_{F}=2 \mathrm{~nm}^{-1}$ (red dashed line), and $k_{F}=2.5 \mathrm{~nm}^{-1}$ (green dash-dotted line).

vectors $q$. The nature of the divergence of $Q$ at $q=2 k_{F}$ is quite specific to TIs, since $q=2 k_{F}$ implies backscattering, which is suppressed by the orthogonality of the initial and final spin states.

\section{B. Renormalization of the phonon dispersion}

To investigate the renormalization of the phonon dispersion by the electronic screening, we calculate the real part of polarization operator $\mathcal{P}(q, \omega)$ in the static limit, $\omega \rightarrow 0$. We find that its bare value $P(q, 0)=-k_{F} / 2 \pi \hbar v_{F}$ is relatively large, but it is heavily suppressed by screening in RPA. RPA yields $\left(\tilde{q}=q / 2 k_{F}\right)$

$$
\mathcal{P}(q, 0)=-\frac{k_{F}}{2 \pi \hbar v_{F}} \frac{\mathcal{F}(\tilde{q})}{\tilde{q}},
$$

where $\mathcal{F}(\tilde{q})$ is given by

$$
\mathcal{F}(q)=\frac{\tilde{q}^{2}}{2} \frac{2-\tilde{q} \Theta(\tilde{q}-1) G_{<}(1 / \tilde{q})}{4 \tilde{q}+\alpha_{F S}\left\{2-\tilde{q} \Theta(\tilde{q}-1) G_{<}(1 / \tilde{q})\right\}},
$$

where $G_{<}(x)=x \sqrt{1-x^{2}}-\arccos x$ and $\Theta$ is the step function. For $\tilde{q} \ll 1, \mathcal{F}(q) \approx \frac{\tilde{q}^{2}}{2}\left[\alpha_{F S}+2 \tilde{q}\right]^{-1}$.

The correction to the phonon dispersion, $\Delta \omega_{q}=$ $|M(q)|^{2} \mathcal{P}(q, 0) / \hbar$, gives the renormalization

$$
\omega_{q}=\omega_{q}^{(0)}\left[1-\frac{K\left(k_{F}\right)}{4} \Lambda_{l}^{2}(q) \mathcal{F}(q)\right],
$$

which is plotted in Fig. 2(a) versus $q$ for $\alpha=35 \mathrm{eV}$ and two values of $k_{F}=1 \mathrm{~nm}^{-1}$ (solid line) and $2.5 \mathrm{~nm}^{-1}$ (dashed line). The inset shows that a renormalization of the phonon velocity occurs only for unrealistically large values of the $e$-ph coupling $\alpha=105 \mathrm{eV}$ (black solid line), $210 \mathrm{eV}$ (red dashed line), and $227.5 \mathrm{eV}$ (green dash-dotted line). However, the dispersion is quite sensitive to changes in the $q$ dependence of the structure factor. This can be seen from the dash-dotted curve in the main panel, which is plotted for $\alpha=35 \mathrm{eV}$ and $k_{F}=1 \mathrm{~nm}^{-1}$, by setting a flat structure factor $\Lambda_{l}(q)=1$.

In wide-gap TIs, the spatial decay of the electronic surface states and of the Rayleigh modes can occur on comparable scales, leading to $\Lambda_{l}(q) \approx 1$. In this case, the softening of 


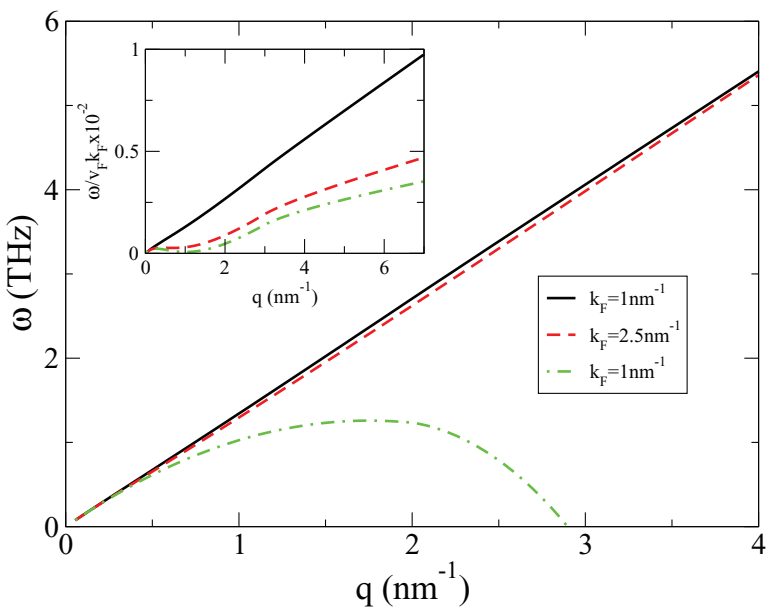

(a)

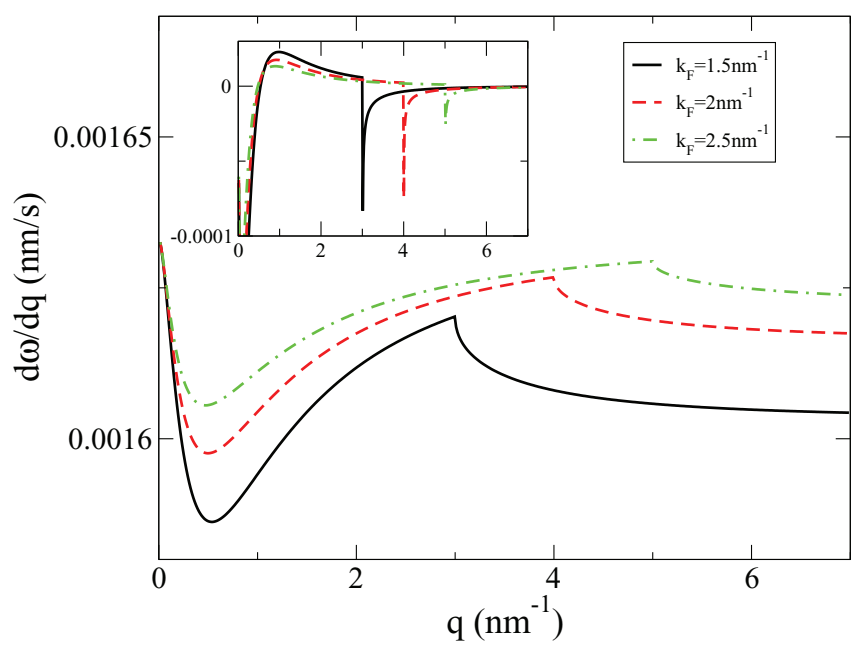

(b)

FIG. 2. (Color online) (a) Plot of the phonon dispersion $\omega_{q}$ given by Eq. (17), for $\alpha=35 \mathrm{eV}$. The solid line and the dashed line are for $k_{F}=1 \mathrm{~nm}^{-1}$ and $k_{F}=2.5 \mathrm{~nm}^{-1}$, respectively. The dash-dotted curve is obtained for $k_{F}=1 \mathrm{~nm}^{-1}$ by setting $\Lambda_{l}(q)=1$. (Inset) The phonon frequency at $k_{F}=1.5 \mathrm{~nm}^{-1}$ (in units $v_{F} k_{F}$ ) versus $q$ for different values of the strength of the deformation potential $\alpha=$ $105 \mathrm{eV}$ (black solid line), $210 \mathrm{eV}$ (red dashed line), and $227.5 \mathrm{eV}$ (green dash-dotted line). (b) Plot of the first derivative of $\omega_{q}$ versus $q$ for $\alpha=35 \mathrm{eV}$ and various $k_{F}$ values. The discontinuity at $q=2 k_{F}$ signals the Kohn anomaly. The inset shows the corresponding marked discontinuities in the second derivative.

the mode could give rise to a lattice instability at some finite wave vector $q_{c}$. This critical value of $q$ depends on material parameters, such as the deformation potential and the sound velocity, and it is also a function of the doping: $q_{c}$ decreases with increasing $k_{F}$, eventually reaching a constant value $q_{c}^{*}$ fixed by the coupling constant $K\left(k_{F}\right)$,

$$
q_{c}^{*}=4 k_{F} \sqrt{\frac{\alpha_{F S}}{K\left(k_{F}\right)}} .
$$

However, the formation of a scalar potential superlattice would weaken the screening and eventually compete with the occurrence of the instability itself. This is because a possible reconstruction would reduce heavily the electronic density of states at $q_{c}$, assumed to be close to $k_{F}$ (see Refs. 15-17 for similar effects in graphene). A lattice instability in wide-gap TIs, induced by the Rayleigh mode at momentum $q_{c} \neq k_{F}$ should be measurable in STM experiments ${ }^{17}$ and would also modify the electronic transport properties.

A signature of the absence of the backscattering induced by the topological protection of the surface states shows up in a milder Kohn anomaly at $q=2 k_{F}$, with respect to the one typical of a conventional 2D electron gas. The nonanalyticity is not recognizable in the dispersion itself, but it appears as a kink in the first derivative, as shown in Fig. 2(b) for three values of $k_{F}$ and $\alpha=35 \mathrm{eV}$. A sharp singularity only develops in the second derivative of the phonon dispersion, as shown in the inset of Fig. 2(b).

\section{EFFECTS OF PHONONS ON ELECTRONS}

We now consider the renormalization of the bare $e-e$ interaction $v_{q}$ by the $e$-ph interaction. The screened $e$-e potential takes the form $V(q, \omega)=\tilde{v}(q, \omega) / \epsilon(q, \omega)$, involving the propagator of the acoustic Rayleigh mode, $D^{(0)}(q, \omega)$,

$$
\tilde{v}(q, \omega)=v_{q}+|M(q)|^{2} D^{(0)}(q, \omega),
$$

and the dielectric function

$$
\frac{\epsilon(q, \omega)}{\epsilon}=1-\tilde{v}(q, \omega) P(q, \omega) .
$$

The static limit $\epsilon(q, 0)$ diverges for $q \rightarrow 0$ due to the metallic nature of the system. The zeros of $\epsilon(q, \omega)$ determine the plasmon dispersion relation. The corrections to the bare plasmon dispersion,

$$
\omega_{p}^{(0)}(q)=\left(\alpha_{F S} \frac{v_{F}^{2} k_{F}}{4 \pi} q\right)^{1 / 2},
$$

are quite small, as can be checked by using the approximate form of the polarization operator $P(q, \omega) \approx \mu q^{2} / 4 \pi \hbar^{2} \omega^{2}$, which is adequate in the limit

$$
1 \ll \frac{\hbar \omega}{\hbar v_{F} q} \ll \frac{\mu}{\hbar v_{F} q} .
$$

The influence of the $e$-ph coupling on the $e$ - $e$ interaction can be found only at low $\omega$ values, when the transferred momentum matches the phonon dispersion. A color plot of the effective interaction at small $\omega$ 's, in the plane $\omega / q$, is shown in Fig. 3(a). We have plotted the dimensionless product $\rho\left(E_{F}\right) V(q, \omega)$, where $\rho\left(E_{F}\right)=k_{F} / 2 \pi \hbar v_{F}$ is the density of states at the Fermi level, per spin direction. A cross section of the plot, marked by the black line in Fig. 3(a) (at $\omega / v_{F} k_{F}=0.005$ ), is reported in Fig. 3(b). The plot shows the sharp change of sign of the interaction in crossing the phonon dispersion.

It is interesting to inquire whether an instability to surface superconductivity in a $\mathrm{TI}$ as $\mathrm{Bi}_{2} \mathrm{Se}_{3}$ or $\mathrm{Bi}_{2} \mathrm{Te}_{3}$ could be triggered by the $e$-ph interaction mediated by the Rayleigh mode. However, the pairing should involve some bulk electron density at the Fermi level, which can be due to impurity bands or doping. In fact, according to our results, the $e$-ph interaction becomes relevant only at $q<\Delta / \hbar v_{F}$, but at these $q$ vectors the Rayleigh mode extends far from the boundary. We can model the pairing interaction in the usual weak-coupling form, 


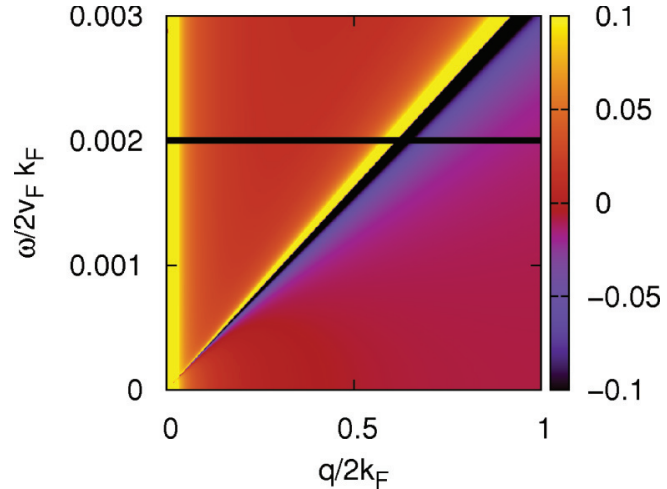

(a)

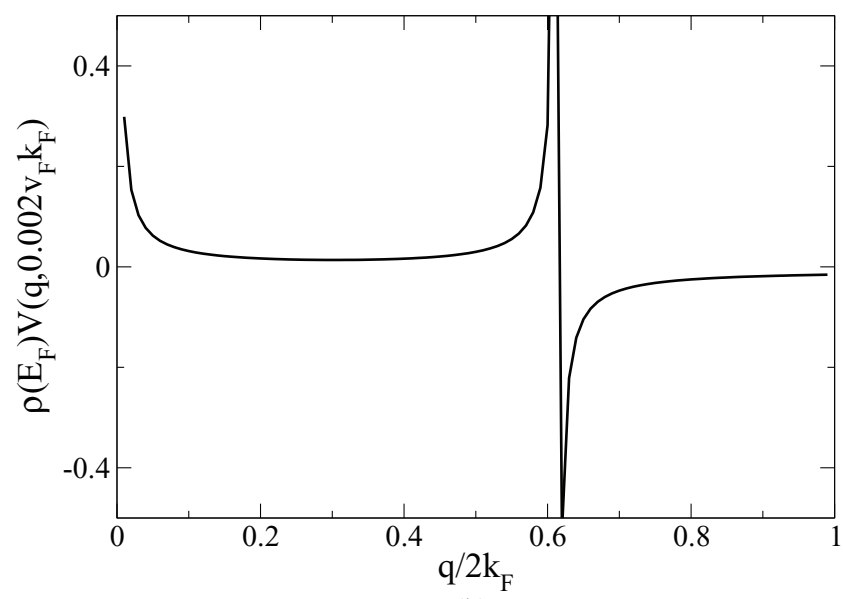

(b)

FIG. 3. (Color online) (a) Color plot of the dimensionless quantity $\rho\left(E_{F}\right) V(q, \omega)$ for $k_{F}=1 \mathrm{~nm}^{-1}$ in the $q / \omega$ plane $(\alpha=35 \mathrm{eV})$. The horizontal black line at $\omega / v_{F} k_{F}=0.002$ marks a cross section of the plot that is drawn separately in the panel (b).

which is appropriate for a conventional phase transition in the bulk material. The critical temperature $T_{c} \sim 1.1 \hbar \omega_{D} e^{-1 /|\lambda|}$ is characterized by the pairing parameter $\lambda<0$. The latter can be estimated as

$$
\lambda \sim \frac{2 \rho\left(E_{F}\right)}{\pi} \int_{0}^{\pi} d \theta \quad V\left(2 k_{F} \sin \frac{\theta}{2}, 0\right) \cos ^{2} \frac{\theta}{2} .
$$

Here the cosine factor accounts for the chirality of the surface electronic states. Since the phonon frequency is very low, we rely on the static value of the interaction only. The product $\rho\left(E_{F}\right) V\left(2 k_{F} \sin \theta / 2,0\right)$ is plotted in Fig. 4 versus the scattering angle $\theta$ for various values of $k_{F}$. Increasing $k_{F}$, there is an increase of the negative values of the interaction. Indeed, the Coulomb repulsion becomes smaller than the phonon-mediated attractive interaction when $k_{F}$ increases, according to the rough estimate

$$
\frac{e^{2}}{\epsilon_{0} k_{F}} \ll \frac{\alpha^{2} C k_{F}}{2 \rho_{M} \bar{c}^{2}},
$$

where $\bar{c}=c_{l}^{2} / c_{R}$. The parameter $\lambda$ is plotted in the inset of Fig. 4 versus $k_{F}$. In terms of the mass in the unit cell $M_{u c}$, the parameter $\lambda$ in the attractive range is approximately

$$
|\lambda| \approx \frac{\alpha^{2} C k_{F} a}{M_{u c} \bar{c}^{2} \mu},
$$

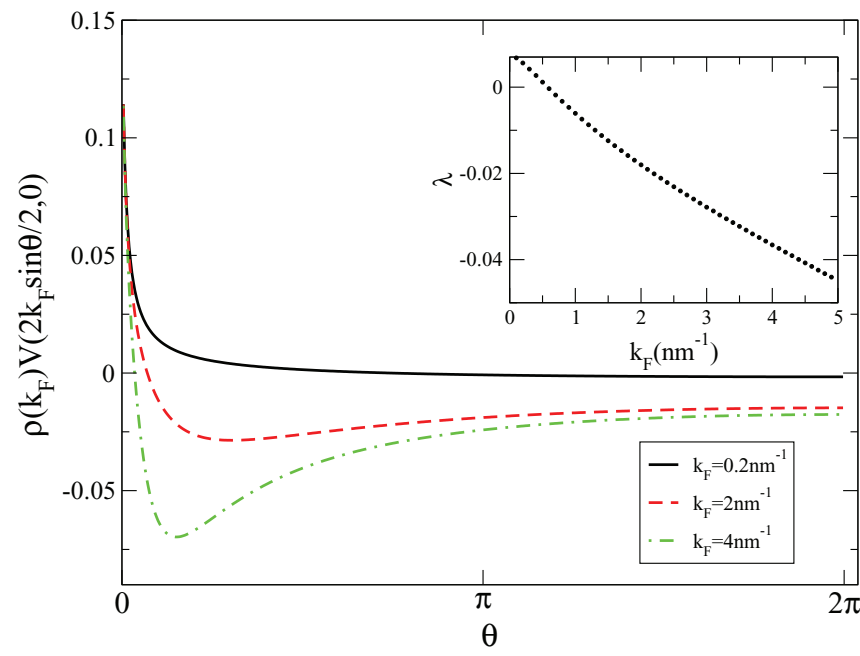

FIG. 4. (Color online) Plot of the dimensionless product $\rho\left(E_{F}\right) V\left(2 k_{F} \sin \theta / 2,0\right)$ of Eq. (23) versus the scattering angle $\theta$ for various values of $k_{F}(\alpha=35 \mathrm{eV})$. The inset shows the dependence of the pairing coupling strength $\lambda$ versus $k_{F}$.

where $a$ is the lattice constant. For $\mu \approx 0.1 \mathrm{eV}$ and $k_{F} \sim$ $1 \mathrm{~nm}^{-1}\left(k_{F} a \approx 0.1\right)$ is $|\lambda| \approx 10^{-2}$. We conclude that not even if $k_{F}$ is large, the $e$-ph interaction driven by the Rayleigh mode can be sizable enough to mediate the superconducting pairing correlations. This seems to be confirmed in the case of $\mathrm{Bi}_{2} \mathrm{Te}_{3}$, which does not become superconducting with $\mathrm{Cu}$ doping. ${ }^{18}$

\section{CONCLUSIONS}

There has been great excitement over the prediction that TIs display protected helical states at the boundaries with Dirac dispersion also in view of the possible applications to spintronics ${ }^{19}$ and to the fabrication of quantum information devices. Nevertheless, questions arise on how robust the protection is when long-range perturbations occur. Electron-phonon scattering could be one of thesources of disruption of this remarkable property of the electronicsurface states. Phonon spectra have recently been measured and it appearsas if the expected acoustical Rayleigh surface mode is absent. ${ }^{4}$ One of the possible explanations for its absence is a strong renormalization of the mode due to the $e$-ph interaction. We have explored this possibility using a continuum longwavelength approach and RPA electronic screening. The $e$-ph matrix element has been evaluated in the ideal case of a flat surface in the absence of defects and with ballistic electron propagation. Its magnitude is strongly dependent on the overlap between the electronic density at the surface and the lattice wave distortions. On the other hand, while the $e$-ph interaction could be larger at small $q$ vectors, the screened electronic response is quite weak for $q \ll 2 k_{F}$, as well as for $q \sim 2 k_{F}$. The influence of the electronic polarization operator is weak at $q \sim 2 k_{F}$ due to the absence of backscattering of the helical states. We conclude that, within our assumptions, we are unable to account for a strong renormalization of the Rayleigh mode. Thus, softening of the mode is also excluded.

We have also checked the $q$ and $\omega$ dependence of the electron-electron interaction within the same approximations. The Rayleigh mode provides a pole in the dielectric response, 
which can change the sign of the $e$ - $e$ interaction. It is known that an increased role of the bulk by doping with intercalated copper drives bulk $\mathrm{Bi}_{2} \mathrm{Se}_{3}$ to superconductivity. This is not the case for the $\mathrm{Bi}_{2} \mathrm{Te}_{3}$ in which copper tends to become substitutional and compensation occurs. ${ }^{18}$ We give an estimate of the possible role of the Rayleigh mode in a conventional weak coupling pairing theory for bulk superconductivity. In the case of BiTe we find that the pairing interaction parameter turns out to be quite small. The case of $\mathrm{Bi}_{2} \mathrm{Se}_{3}$ would be more favorable because, due to the larger bulk gap, the surface electron states are more localized at the boundaries, which enhances the $e$-ph matrix element.

Recently, state-of-the-art ARPES measurements have been reported, ${ }^{20}$ which point to an exceptionally large constant for the coupling to low-lying excitation modes $\lambda \sim 3$ in $\mathrm{Bi}_{2} \mathrm{Se}_{3}$ and $\mathrm{Bi}_{2} \mathrm{Te}_{3}$. These excitations could be the optical mode of surface phonons, the electron-hole pair generation or the predicted electron-plasmon coupling. Remarkably, there is no overall band reconstruction for the Dirac cone down to the lowest temperature. In this work we prove that, within standard approximations, the $e$-ph interaction between the Dirac electrons and the Rayleigh phonon mode cannot be responsible of strong renormalization effects and our estimate for the $e$-ph coupling constant to this mode is $\lambda \sim 0.01$. Our estimate is not necessarily comparable to a generic $e$-ph coupling constant reported in a recent paper, ${ }^{21}$ which is one order of magnitude larger. While the formula used are similar [see our Eq. (25)], the discrepancy can be attributed mostly to the fact that we use the mass in the unit cell $M_{u c}$ in Eq. (25). The unit cell spans three quintuple layers, while the other authors consider just one quintuple layer. As the Rayleigh mode extends rather deep away from the surface at small $q$ vectors, we think that our choice is more appropriate to our case.

\section{ACKNOWLEDGMENTS}

One of us (V.P.) acknowledges useful discussions with E. Cappelluti and P. Lucignano. This work was done with financial support from FP7/2007-2013 under Grant No. 264098-MAMA (Multifunctioned Advanced Materials and Nanoscale Phenomena), MIUR-Italy through Prin-Project 2009 "Nanowire high critical temperature superconductor field-effect devices," as well as the Helmholtz Virtual Insitute "New States of Matter." V.P. and F.G. acknowledge financial support from MINECO, Spain, through Grant No. FIS201123713, and the European Union, through Grant No. 290846.

\section{APPENDIX A: MATRIX ELEMENTS OF THE $e$-ph INTERACTION}

The matrix elements of the $e$-ph interaction appearing in Eq. (6) require that the contraction of the strain tensor $\left(\partial_{i} U_{j}+\partial_{j} U_{i}\right) / 2$ with appropriate $4 \times 4$ matrix $A_{i j}$ is invariant with respect to the symmetry operations of the little groups ${ }^{11}$ preserving the wave vectors of the electrons and phonons, respectively. Long-wavelength phonons have momenta close to the $\Gamma$ point and thus the little group for a generic $\vec{q}$ is the space group of the crystal. In TIs as $\mathrm{Bi}_{2} \mathrm{Se}_{3}$ the surface states are close to the $\Gamma$ point as well, so that the little group
TABLE I. Basis in the space of $4 \times 4$ matrices.

\begin{tabular}{lccccc}
\hline \hline Matrix & $C_{6}$ & $\mathcal{T}$ & Matrix & $C_{6}$ & $\mathcal{T}$ \\
\hline $\mathbb{I}$ & $\mathrm{Y}$ & $\mathrm{Y}$ & $i \gamma_{2} \gamma_{5}$ & & \\
$\gamma_{0}$ & $\mathrm{Y}$ & $\mathrm{Y}$ & $i \gamma_{3} \gamma_{5}$ & $\mathrm{Y}$ & \\
$\gamma_{1}$ & & & $i \Sigma_{01}$ & & $\mathrm{Y}$ \\
$\gamma_{2}$ & & & $i \Sigma_{02}$ & & $\mathrm{Y}$ \\
$\gamma_{3}$ & $\mathrm{Y}$ & & $i \Sigma_{03}$ & $\mathrm{Y}$ & $\mathrm{Y}$ \\
$\gamma_{5}$ & $\mathrm{Y}$ & & $\Sigma_{12}$ & $\mathrm{Y}$ & \\
$\gamma_{0} \gamma_{5}$ & $\mathrm{Y}$ & $\mathrm{Y}$ & $\Sigma_{13}$ & & \\
$i \gamma_{1} \gamma_{5}$ & & & $\Sigma_{23}$ & & \\
\hline \hline
\end{tabular}

of the surface states is again the space group of the lattice, as for the phonons. In the case of $\mathrm{Bi}_{2} \mathrm{Se}_{3}$ the space group is $G=\left\{C_{6}, \mathcal{I}, \mathcal{T}\right\}$, where $\mathcal{I}$ is the space inversion and $\mathcal{T}$ is the time reversal. Let the orbital $\otimes$ spin space be addressed by the Pauli matrices $\vec{\tau}$ and $\vec{\sigma}$, respectively. The Dirac matrices are $\gamma_{0}=i \mathbb{I} \otimes \tau_{z}, \gamma_{i}=\sigma_{i} \otimes \tau_{x}(i=1, \ldots, 3)$, where $\mathcal{I}$ is the identity matrix. In the first and the fourth column of Table I we enumerate all the matrices $\Gamma_{v}$ that can be built in the given space. Besides the identity and $\gamma_{5}=i \alpha_{0} \gamma_{1} \gamma_{2} \gamma_{3}=-\mathbb{I} \otimes \tau_{y}$, there are the four matrices $\gamma_{0} \gamma_{5}=\mathbb{I} \otimes \tau_{x}, \gamma_{i} \gamma_{5}=-i \sigma_{i} \otimes \tau_{z}$ and the six matrices $\Sigma_{\mu \nu}=i / 2\left[\gamma_{\mu}, \gamma_{\nu}\right]$ given by

$$
\Sigma_{0 i}=-i \sigma_{i} \otimes \tau_{y}, \quad \Sigma_{i j}=\epsilon_{i j k} \sigma_{k} \otimes \mathbb{I}
$$

(with $i<j$ ). These 15 matrices, plus the identity matrix, make a basis in the space of $4 \times 4$ matrices $\left\{\Gamma_{i}\right\}$ explicitly written in Table I. The label $Y$ in the $C_{6}$ and $\mathcal{T}$ columns of the Table marks the matrices which are conserved by the symmetry operations $C_{6}$ or $\mathcal{T}$

From Table I it is possible to extract four constant vectors, reported in Table II, which give rise to seven second-rank tensors conserving time reversal: $a_{i} a_{j}, b_{i} b_{j}, b_{i}^{\prime} b_{j}^{\prime}, p_{i} p_{j}, a_{i} b_{j}, b_{i} b_{j}^{\prime}, a_{i} b_{j}^{\prime}$. The tensors $a_{i} b_{j}$ and $a_{i} b_{j}^{\prime}$ must be dropped because they break inversion symmetry. The first surviving tensors are identical, since

$$
\Sigma_{\mu \nu} \Sigma_{\mu \eta}=\gamma_{\nu} \gamma_{\eta}, \quad\left(i \gamma_{\mu} \gamma_{5}\right)\left(i \gamma_{\nu} \gamma_{5}\right)=\gamma_{\mu} \gamma_{\nu},
$$

so that we are left just with the two independent bilinears:

$$
\begin{aligned}
& A_{i j} \equiv a_{i} a_{j}=\left(\sigma_{i} \otimes \tau_{x}\right)\left(\sigma_{j} \otimes \tau_{x}\right)=\delta_{i j} \mathbb{I} \otimes \mathbb{I}+i \epsilon_{i j k} \sigma_{k} \otimes \mathbb{I}, \\
& B_{i j} \equiv b_{i} b_{j}^{\prime}=\left(\sigma_{i} \otimes \mathbb{I}\right)\left(\sigma_{j} \otimes \tau_{z}\right)=\delta_{i j} \mathbb{I} \otimes \tau_{z}+i \epsilon_{i j k} \sigma_{k} \otimes \tau_{z} .
\end{aligned}
$$

Contraction of each of these matrices with the symmetric strain tensor cancels the antisymmetric part of both bilinears. We ignore the perturbative matrix elements arising from $B_{i j}$ because they would induce changes in the bulk gap

TABLE II. Matrices used to build time reversal and parity invariant electron-phonon interaction.

\begin{tabular}{lcc}
\hline \hline & TR & I \\
\hline $\boldsymbol{a}=\left(\gamma_{1}, \gamma_{2}, \gamma_{3}\right)$ & -1 & -1 \\
$\boldsymbol{b}=\left(\Sigma_{23}, \Sigma_{31}, \Sigma_{12}\right)$ & -1 & +1 \\
$\boldsymbol{p}=\left(i \Sigma_{01}, i \Sigma_{02}, i \Sigma_{03}\right)$ & +1 & -1 \\
$\boldsymbol{b}^{\prime}=\left(i \gamma_{1} \gamma_{5}, i \gamma_{2} \gamma_{5}, i \gamma_{3} \gamma_{5}\right)$ & -1 & +1 \\
\hline \hline
\end{tabular}


of the material and we get the final result of Eq. (6) with $A_{i j}=\delta_{i j} \mathbb{I} \otimes \mathbb{I}$.

We stress that this result leads to an $e$-ph coupling matrix, which is diagonal in the helicity of the Dirac electrons. This is at difference with the case of graphene which was discussed in Ref. 22. The $e$-ph coupling matrix for Dirac electrons in graphene, close to the $K, K^{\prime}$ points, is nondiagonal, due to the reduced symmetry of the $K, K^{\prime}$ point, with respect to the $\Gamma$ point. The little group at the $K$ point of the graphene Brillouin zone is generated by $\left\{C_{3}^{+}, \sigma_{v 1}, C_{2}\right\}$, where $C_{3}^{+}$is the generator of rotations around the $z$ axis (the graphene sheet is supposed to lay in the $x y$ plane), $\sigma_{v 1}$ is a reflection in the $x z$ plane, and $C_{2}$ is a binary rotation around the $z$ axis. The representation of the generators acting on the electron states in the sublattice basis are

$$
\begin{gathered}
T\left(C_{3}\right)=e^{i \sigma_{z} 2 \pi / 3}=\left(\begin{array}{cc}
e^{i 2 \pi / 3} & 0 \\
0 & e^{-i 2 \pi / 3}
\end{array}\right), \\
T\left(\sigma_{v 1}\right)=\sigma_{x}, \quad T\left(C_{2}\right)=\mathcal{I}_{2 D},
\end{gathered}
$$

where $\mathcal{I}_{2 D}$ is the in plane space inversion. The action of the reflection $\sigma_{v 1}$ on momenta is $\sigma_{v 1} q=q^{*}$, where $q=q_{x}+i q_{y}$. Both $C_{2}$ and the time reversal $\mathcal{T}$ map $K$ into $K^{\prime}$ by reversing the direction of momenta. Still the combination of the two, $C_{2} \mathcal{T}$, maps states of one cone in states of the same cone but it changes the two atoms in the unit cell; i.e., it acts like $\sigma_{x} \mathcal{K}$, where $\mathcal{K}$ is the complex conjugation coming from the time reversal.

If we write the $e$-ph Hamiltonian as

$$
H_{e-\mathrm{ph}}=\sum_{s, s^{\prime}, k, q} H_{s s^{\prime}}(k, q, Q) c_{s}^{\dagger}(k+q / 2) c_{s^{\prime}}(k-q / 2),
$$

where $Q$ is the amplitude of the acoustical phonon mode, the $\mathrm{C}_{2} \mathcal{T}$ invariance requires the simplified matrix form

$$
H(k, q, Q)=\left(\begin{array}{cc}
\alpha(k, q, Q) & \beta(k, q, Q) \\
\beta^{*}(k, q, Q) & \alpha^{*}(k, q, Q)
\end{array}\right),
$$

with nonvanishing off-diagonal elements. $\alpha$ and $\beta$ are restricted further, by using the remaining symmetries. By applying the rotation invariance, the functions $\alpha$ and $\beta$ satisfy the relations $\left(\omega=e^{i 2 \pi / 3}\right)$

$$
\begin{aligned}
& \alpha\left(\omega k, \omega q, C_{3}^{+} Q\right)=\alpha(k, q, Q), \\
& \beta\left(\omega k, \omega q, C_{3}^{+} Q\right)=\omega^{*} \beta(k, q, Q),
\end{aligned}
$$

while the invariance with respect to the reflection $\sigma_{v 1}$ poses the conditions

$$
\begin{aligned}
& \alpha\left(k^{*}, q^{*}, \sigma_{v 1} Q\right)=\alpha^{*}(k, q, Q), \\
& \beta\left(k^{*}, q^{*}, \sigma_{v 1} Q\right)=\beta^{*}(k, q, Q) .
\end{aligned}
$$

In the case of the 3D TI, the little group at the projection of the $\Gamma$ point onto the surface, in the continuum limit, includes all rotations around the $z$ axis plus the inversion symmetry and time reversal. The presence of a single Dirac cone, in particular, avoids the use of off-diagonal symmetry operations like $C_{2} \mathcal{T}=\sigma_{x} \mathcal{K}$. As proved in the first part of this appendix, which deals with the full $4 \times 4$ orbital $\otimes$ spin space, the $e$-ph interaction has to be proportional to the identity matrix in order to commute with all the symmetry operations.

\section{APPENDIX B: THE ELECTRONIC POLARIZATION $P$ FOR TWO-DIMENSIONAL DIRAC ELECTRONS}

In this appendix we collect the main results of the derivation of the zero-temperature electronic polarization calculated for 2D electrons having a Dirac energy dispersion. ${ }^{14}$ At zero temperature the polarization is written as a $P_{0}$ term, having $\mu=0$, and a term $\Delta P$ depending on the chemical potential

$$
P(k, \omega)=P_{0}+\Delta P .
$$

Here we use dimensionless variables $x=q / 2 k_{F}$ and $y=$ $\omega / 2 v_{F} k_{F}$ and $P \rightarrow P / 2 \pi v_{0}$, where $v_{0}=\mu / 2 \pi \hbar^{2} v_{F}^{2}$ is the electronic density of states at the Fermi level. While $P_{0}$ reads simply

$$
P_{0}=-i \frac{1}{8} \frac{x^{2}}{\sqrt{y^{2}-x^{2}}}
$$

the term depending on $\mu$ is

$$
\begin{aligned}
\Delta P= & -\frac{1}{2 \pi}+\frac{1}{8 \pi} \frac{x^{2}}{\sqrt{y^{2}-x^{2}}} \\
& \times\left\{G\left(\frac{y+1}{x}\right)-\Theta\left(\frac{1-y}{x}-1\right)\left[G\left(\frac{1-y}{x}\right)-i \pi\right]\right. \\
& \left.+-\Theta\left(\frac{y-1}{x}+1\right) G\left(\frac{y-1}{x}\right)\right\} .
\end{aligned}
$$

Here the function $G(x)$ is

$$
G(x)=x \sqrt{x^{2}-1}-\ln \left(x+\sqrt{x^{2}-1}\right) .
$$

In limits $x \ll y \ll 1$ the polarization reduces to

$$
P=\frac{1}{\pi} \frac{x^{2}}{y^{2}}
$$

When $y<x$ three cases can be distinguished.

(i) $x<1-y$. Only the first $\Theta$ is different from zero. The real and the imaginary part of the polarization are

$$
\begin{aligned}
& \operatorname{Re}[\Delta P]=-\frac{1}{2 \pi}, \\
& \operatorname{Im}[\Delta P]=-\frac{1}{8 \pi} \frac{x^{2}}{\sqrt{\left|y^{2}-x^{2}\right|}}\left\{G\left(\frac{y+1}{x}\right)-G\left(\frac{1-y}{x}\right)\right\} .
\end{aligned}
$$

(ii) $1-y<x<1+y$. The first $\Theta$ function vanishes, while the second one is different from zero. The real and imaginary part of the polarization are

$$
\begin{aligned}
& \operatorname{Re}[\Delta P]=-\frac{1}{2 \pi}+\frac{1}{8 \pi} \frac{x^{2}}{\sqrt{\left|y^{2}-x^{2}\right|}} G_{<}\left(\frac{1-y}{x}\right), \\
& \operatorname{Im}[\Delta P]=\frac{1}{8 \pi} \frac{x^{2}}{\sqrt{\left|y^{2}-x^{2}\right|}} G\left(\frac{y+1}{x}\right),
\end{aligned}
$$

where $G_{<}(x)=x \sqrt{1-x^{2}}-\arccos x$.

(iii) $x>1+y$. The polarization is real, since both the $\Theta$ 's are zero, but $G(1+y / x)$ is complex,

$$
\operatorname{Re}[\Delta P]=-\frac{1}{2 \pi}+\frac{1}{8 \pi} \frac{x^{2}}{\sqrt{\left|y^{2}-x^{2}\right|}} G_{<}\left(\frac{y+1}{x}\right) .
$$


From this analysis we can conclude that when $\omega<v_{F} q$ the imaginary part of $P(q, \omega)$ is finite for values of the wave vector $q$ up to $1+y$, i.e., $2 k_{F}+\omega / v_{F}$, indicating that the phonon has a finite inverse lifetime in this sector of the $(q, \omega)$ plane.

\section{APPENDIX C: CONDITIONS FOR THE EXISTENCE OF THE RAYLEIGH MODE IN A SLAB GEOMETRY}

Questions arise whether a surface layer with softer elastic constants, attached to a more rigid background, still supports the Rayleigh mode. Below we answer positively this question by discussing the existence conditions within linear elasticity theory in the limit of a thin surface layer (hereafter defined "the slab"). Let us consider a slab with elastic constants $\lambda_{1}, \mu_{1}$, with thickness $h$, rigidly attached to a semi-infinite medium occupying the region $z<0$, with constants $\lambda_{2}, \mu_{2}$. Both the slab and the bulk are considered homogeneous elastic media described by the stress tensor

$$
\begin{aligned}
\sigma_{i j} & =\frac{E}{1+\sigma}\left(u_{i j}+\frac{\sigma}{1-2 \sigma} u_{l l} \delta_{i j}\right) \\
& =2 \rho\left[c_{t}^{2} u_{i j}+\left(c_{l}^{2}-2 c_{t}^{2}\right) u_{l l} \delta_{i j}\right] .
\end{aligned}
$$

Boundary conditions are given by

(i) free surface condition at $z=h$,

$$
\left.\sigma_{1} \cdot n_{z}\right|_{z=h}=0
$$

(ii) continuity of stress at $z=0$,

$$
\left.\sigma_{1} \cdot n_{z}\right|_{z=0}=\left.\sigma_{2} \cdot n_{z}\right|_{z=0}
$$

(iii) continuity of displacement at $z=0$,

$$
\left.u_{1}\right|_{z=0}=\left.u_{2}\right|_{z=0} .
$$

In the geometry described above two different polarizations are possible for surface phonons: Rayleigh modes, polarized in the $x z$ plane, and the Love waves, polarized in the $x y$ plane. We consider only the Rayleigh mode here, as a discussion on Love can be found, for example, in Ref. 23.

To write the general ansatz on the displacement vector we introduce the function $\phi(\mathbf{r})$ and the vector function $\chi(\mathbf{r})$, so that the longitudinal and the transverse parts of $\mathbf{u}$ read

$$
\mathbf{u}_{L}(\mathbf{r})=\nabla \phi, \quad \mathbf{u}_{T}(\mathbf{r})=\nabla \times \chi .
$$

The function $\phi$ and each component of $\chi$ satisfy the wave equation with velocity of propagation $c_{l}$ and $c_{t}$, respectively. The most general forms of these functions giving surface waves polarized in the $x z$ plane of the slab are

$$
\begin{aligned}
& \phi=\left(A \sinh \lambda_{l} z+B \cosh \lambda_{l} z\right) e^{i(k x-\omega t)}, \\
& \chi=\hat{e}_{y}\left(C \sinh \lambda_{t} z+D \cosh \lambda_{t} z\right) e^{i(k x-\omega t)} .
\end{aligned}
$$

Correspondingly, the functions $\phi$ and $\chi$ for the background are

$$
\phi=E e^{\lambda_{l} z} e^{i(k x-\omega t)}, \quad \chi=\hat{e}_{y} F e^{\lambda_{t} z} e^{i(k x-\omega t)} .
$$

The solution of this system of six equations in the six unknowns given by Eqs. (C2), (C3), and (C4) exists provided the determinant of the coefficient matrix vanishes. In principle, the vanishing of the determinant defines the dispersion relation for the surface waves. In this case, the secular equation will be of the 12th degree, and some simplification is needed to understand the physics in a faster and clearer way. The first step is to consider a thin slab, whose thickness is negligible compared to the background. In this case, one can take only the zeroth approximation for displacement vector in the slab. This approximation is possible since, in the slab, there is a combination of hyperbolic functions in the definition of $\mathbf{u}$. In this approximation the system becomes

$$
\begin{aligned}
2 i k \lambda_{l 1} A-\left(k^{2}+\lambda_{t}^{2}\right) D & =0, \\
{\left[c_{l 1}^{2} \lambda_{l 1}^{2}-\left(c_{l 1}^{2}-2 c_{t 1}^{2}\right) k^{2}\right] B+2 i k \lambda_{t 1} c_{t 1}^{2} C } & =0 \\
{\left[2 i k \lambda_{l 2} E-\left(k^{2}+\lambda_{t 2}^{2}\right) F\right] } & =0 \\
{\left[c_{l 2}^{2} \lambda_{l 2}^{2}-\left(c_{l 2}^{2}-2 c_{t 2}^{2}\right) k^{2}\right] E+2 i k \lambda_{t 1} c_{t 1}^{2} F } & =0, \\
i k B-\lambda_{t 1} C & =i k E-\lambda_{t 2} F, \\
\lambda_{l 1} A+i k D & =\lambda_{l 2} E+i k F .
\end{aligned}
$$

The structure of system (C8) enlightens the physics of surface states of this composite material. The third and the fourth equations for $E$ and $F$ above coincide with the system defining Rayleigh waves at the interface between the slab and the background. ${ }^{10}$ Taking a nontrivial solution for granted, the penetration of this mode leads to the definition of Rayleigh waves at the interface between the slab and the vacuum obtained from the remaining equations.

In particular, we are left with the inhomogeneous system of four equations in the four unknowns $A, B, C, D$ :

$$
\begin{aligned}
2 i k \lambda_{l 1} A-\left(k^{2}+\lambda_{t}^{2}\right) D & =0, \\
{\left[c_{l 1}^{2} \lambda_{l 1}^{2}-\left(c_{l 1}^{2}-2 c_{t 1}^{2}\right) k^{2}\right] B+2 i k \lambda_{t 1} c_{t 1}^{2} C } & =0, \\
i k B-\lambda_{t 1} C & =M_{1}, \\
\lambda_{l 1} A+i k D & =M_{2},
\end{aligned}
$$

where $M_{1}$ and $M_{2}$ are assumed to be given quantities, once the homogeneous system for $E, F$ has been solved. They involve the ratio between the velocity of Rayleigh waves $c_{R}$ and the transverse velocity in the bulk, $\xi_{2}=c_{R} / c_{t 2}$. In this case, the determinant $\mathcal{D}$ of the matrix for the coefficients $A, B, C, D$ has to be different from zero,

$$
\mathcal{D}=c_{l 1}^{2} \lambda_{l 1} \lambda_{t 1}\left(k^{2}-\lambda_{t 1}^{2}\right)\left(\lambda_{l 1}^{2}-k^{2}\right),
$$

provided that, or, using the definition of the $\lambda$ 's given in the main text $\lambda_{l}=\sqrt{1-c_{R} / c_{l}}$ and $\lambda_{t}=\sqrt{1-c_{R} / c_{t}}$,

$$
\begin{gathered}
\mathcal{D}=-c_{l 1}^{2} \sqrt{1-\gamma^{2} \xi^{2}} \sqrt{1-\xi^{2}} k^{6} \xi^{4} \gamma^{2}, \\
\xi_{2}=\frac{c_{R}}{c_{t 2}}=\frac{c_{R}}{c_{t 1}} \frac{c_{t 1}}{c_{t 2}}=\xi_{1} \frac{c_{t 1}}{c_{t 2}} .
\end{gathered}
$$

The determinant $\mathcal{D}$ can be nonzero provided $0 \neq \xi_{1}<1$ and therefore the system of Eq. (C9) has a nontrivial solution. There are some restrictions, though, on the elastic parameters in the two materials, stemming from $\xi_{1}<1$ :

$$
\xi_{2} \frac{c_{t 2}}{c_{t 1}}<1 \Rightarrow \frac{c_{t 2}}{c_{t 1}}<\frac{1}{\xi_{2}} .
$$


${ }^{1}$ H. Zhang, C.-X. Liu, X.-L. Qi, X. Dai, Z. Fang, and S.-C. Zhang, Nat. Phys. 5, 438 (2009).

${ }^{2}$ M. Z. Hasan and C. L. Kane, Rev. Mod. Phys. 82, 3045 (2010).

${ }^{3}$ S. Giraud and R. Egger, Phys. Rev. B 83, 245322 (2011).

${ }^{4}$ X. Zhu, L. Santos, R. Sankar, S. Chikara, C. Howard, F. C. Chou,

C. Chamon, and M. El-Batanouny, Phys. Rev. Lett. 107, 186102 (2011).

${ }^{5}$ X. Zhu, L. Santos, C. Howard, R. Sankar, F. C. Chou, C. Chamon, and M. El-Batanouny, Phys. Rev. Lett. 108, 185501 (2012).

${ }^{6}$ Z.-H. Pan, A. V. Fedorov, D. Gardner, Y. S. Lee, S. Chu, and T. Valla, Phys. Rev. Lett. 108, 187001 (2012).

${ }^{7}$ R. L. V. Sandomirsky, A. V. Butenko, and Y. Schlesinger, J. Appl. Phys. 90, 2370 (2001).

${ }^{8}$ D. Greenaway and G. Harbeke, J. Phys. Chem. Solids 26, 1585 (1965).

${ }^{9}$ H. Ezawa, Ann. Phys. 67, 438 (1971).

${ }^{10}$ L. D. Landau and E. M. Lifshitz, Theory of Elasticity (Pergamon Press, Oxford, UK, 1975).

${ }^{11} \mathrm{G}$. Lyubarskii, The Application of Group Theory (Pergamon Press, Oxford, UK, 1960).

${ }^{12}$ B.-L. Huang and M. Kaviany, Phys. Rev. B 77, 125209 (2008).
${ }^{13}$ G. D. Mahan, Many-Particle Physics (Plenum Press, New York, 1990).

${ }^{14}$ B. Wunsch, T. Stauber, F. Sols, and F. Guinea, New J. Phys. 8, 318 (2006).

${ }^{15}$ C.-H. Park, L. Yang, Y.-W. Son, M. L. Cohen, and S. G. Louie, Phys. Rev. Lett. 101, 126804 (2008).

${ }^{16}$ F. Guinea and T. Low, Philos. Trans. R. Soc. A 368, 5391 (2010).

${ }^{17}$ M. Yankowitz, J. Xue, D. Cormode, J. D. Sanchez-Yamagishi, K. Watanabe, T. Taniguchi, P. Jarillo-Herrero, P. Jacquod, and B. J. LeRoy, Nat. Phys. 8, 382 (2012).

${ }^{18}$ Y. Tanaka, K. Nakayama, S. Souma, T. Sato, N. Xu, P. Zhang, P. Richard, H. Ding, Y. Suzuki, P. Das, K. Kadowaki, and T. Takahashi, Phys. Rev. B 85, 125111 (2012).

${ }^{19}$ J. G. Analytis, R. D. McDonald, S. C. Riggs, J.-H. Chu, G. S. Boebinger, and I. R. Fisher, Nat. Phys. 6, 960 (2010).

${ }^{20}$ T. Kondo, Y. Nakashima, Y. Ota, Y. Ishida, W. Malaeb, K. Okazaki, S. Shin, M. Kriener, S. Sasaki, K. Segawa, and Y. Ando, Phys. Rev. Lett. 110, 217601 (2013).

${ }^{21}$ S. Das Sarma and Q. Li, Phys. Rev. B 88, 081404(R) (2013).

${ }^{22}$ J. L. Mañes, Phys. Rev. B 76, 045430 (2007).

${ }^{23}$ J. Pujol, Elastic Wave Propagation and Generation in Seismology (Cambridge University Press, Cambridge, UK, 2003). 